\title{
THE STRONG NORMAL SYSTEM OF SOME COMPACT RIGHT TOPOLOGICAL GROUPS
}

\author{
ZOHREH BAHRAMIAN and ALI JABBARI
}

\begin{abstract}
The aim of the present paper is to characterize the strong normal system of the Ellis groups of a well-known family of dynamical systems on the finite and infinite dimensional tori.

MSC 2010. 43A60.

Key words. Ellis group, strong normal system, CHART group, compact right topological group, Furstenberg-Ellis-Namioka Structure Theorem.
\end{abstract}

\section{REFERENCES}

[1] R. Ellis, Distal transformation groups, Pacific J. Math., 8 (1958), 401-405.

[2] R. Ellis, The Furstenberg structure theorem, Pacific J. Math., 76 (1978), 345-349.

[3] H. Furstenberg, The structure of distal flows, Amer. J. Math., 83 (1963), 477-515.

[4] F. Hahn, Skew product transformations and the algebras generated by $\exp (p(n))$, Illinois J. Math., 9 (1965), 178-190.

[5] A. Jabbari and I. Namioka, Ellis group and the topological center of the flow generated by the map $n \mapsto \lambda^{n^{k}}$, Milan J. Math., 78 (2010), 503-522.

[6] A. Jabbari and H.R.E. Vishki, Skew product dynamical systems, Ellis groups and topological center, Bull. Aust. Math. Soc., 79 (2009), 129-145.

[7] P. Milnes and J. Pym, Haar measure for compact right topological groups, Proc. Amer. Math. Soc., 114 (1992), 387-393.

[8] W.B. Moors and I. Namioka, Furstenberg's structure theorem via CHART groups, Ergodic Theory Dynam. Systems., 33 (2013), 954-968.

[9] I. Namioka, Right topological groups, distal flows and a fixed-point theorem, Math. System Theory, 6 (1972), 193-209.

[10] J. Rautio, Enveloping semigroups and quasi-discrete spectrum, Ergodic Theory Dynam. Systems, 36 (2016), 2627-2660.

[11] M. Zaman-Abadi and A. Jabbari, The Furstenberg-Ellis-Namioka structure theorem on a CHART group, Bull. Iranian Math. Soc., 44 (2018), 409-415.

Received October 27, 2019

Accepted June 2, 2020

The authors thank the referee for his helpful comments and suggestions.

DOI: 10.24193/mathcluj.2021.2.02 
Shahid Bahonar University of Kerman Faculty of Mathematics and Computer Department of Pure Mathematics

Kerman, Iran

E-mail: arshta@yahoo.com

E-mail: jabbari@uk.ac.ir 